1851.

I. Ueber das elektrische Leitungscermögen des Schwefelsilbers und Halbschwefelkupfers; von Dr. Hittorff in Münster.

Zwei wesentlich verschiedene Processe, die gewöhnliche Leitung und die Elektrolyse, verinitteln in den Körpern den Durchgang des galvanischen Stromes. Das Vermögen, die Elektricität durch erstern Vorgang fortzupflanzen, finden wir am stärksten bei den Metallen; nach ihnen soll im Folgenden diese Leitung die metallische heifsen. Die elektrolytische Leitung besitzen eine Anzahl zusanmengeselzter Körper, wenn sie sich in flüssigen Zustande befinden, mag derselbe durch ein Auflösungsmiltel oder durch die Wärme herbeigeführt worden seyn.

Das Leilungsvermögen der Elektrolyte, welche bis jelzt genauer untersucht wurden, beruht nur auf ihrer Zersetzbarkeit; sie besitzen nicht die Fähigkeit, den Strom nach Art der Metalle fortzupflanzen. Dieses zeigt sich näılich, sobald Umstände eintreten, welche die Zerselzung verhindern.

Faraday machte hierauf zucrst mit Bestimmtheit aufmerksam, als er fand, wic die elektrolytischen Flüssigkeiten durch den Uebergang in den festen Aggregatzustand, Iso. latoren werden. Mit der Beweglichkeit ihrer kleinsten Theilchen, der nothwendigen Bedingung für eine Trennung, büfsen sie ihr Leitungsvernögen ein. In auffallender Weise zeigt es ferner der schöne Versuch, den Poggendorff vor einigen Jahren mittheilte'). Daruach werden die ron $\mathrm{Oh}$ m aufgestellten Gesetze der Zweigströme in dem Falle 1) Pogg. Ann. Bd. 64, S. 54.

Poggendorf's Annal. Bd. LXXXIV. 
ungültig, wo ein gerader metallischer Leiter innerhalb eines Elektrolyten sich befindet. Wird nämlich cin Metalldraht in einem Gefälse gerade ausgespannt, so zeigt sich der Widerstand genau derselbe, das Gefüls mag leer oder mit einem Elektrolyten gefüllt seyn. Die Zersetzung des letztern tritt hier nicht ein; er hört damit zugleich auf, Lciter zu seyn. Für obige Behauptung sprechen aufserdem dic zuerst von Pouillet und Jacobi angestellen Vergleiche des Voltameters und magnelischen Galvanometers. Die Menge der ausgeschiedenen Bestandtheile eines Elektrolyten ist genau proportional der Stärke des Stromes, wie sie durch die Ablenkung der Magnetnadel sich kund giebt. In den Fällen endlich, wo Elcktrolyte ganz schwache Ströme ohne Zersetzung zu leiten schienen, indem dic gasförmigen ausgeschiedenen Bestandtheile nicht zu bemerken waren, ergab sich bald bei genauer Untersuchung das Gegentheil. In der Polarisation der Elektroden offenbarten sich dic vermifsten Stoffe.

Einen förmlichen Gegensatz scheinen unsere beiden Leiter in der Art und Weise zu bilden, in der die Temperatur ihr Leitungsvermögen inodificirt. Die Metalle nämlich, welche bis jetzt in dieser Hinsicht untersucht wurden, zeigen alle, wenn die Temperatur steigt, eine Zunahme, dic Elektrolyte dagegen eine Abnahunc des Widerstandes. De la Rive hält diesen Unterschied darin begründet, dals in den Metallen, als ciufachen Körpern, die Erhöhung der Temperatur nur eine physische Wirkung, cin Auseinanderweichen der Theilchen hervorbringt und dadurch den Widerstand vermehrt; während sie auf die Elektrolyte chemisch einwirkt, durch Schwächung der Affinität der Ionen die Zerselzung und damit den Durchgang des Stromes erleichtert. Trotz der grofsen Verschiedenhcit, welche zwischen beiden Vorgängen der Leilung besteht, zeigt sich in den übrigen Beziebungen vollständige Uebereinstimmung. Der Widerstand, den beide Leiter dem Strome bieten, gehorcht demselben Gesetze: er wächst proportional mit der Länge und nimmt ab unit dem Querschnitte. Ebenso ist 
die Wärmemenge, welcbe der Strom in beiden erregt, dieselbe unter gleichen Bedingungen. Denn sie steht bei beiden in einfachen Verbältnisse zum Widerstande des Leiters und wächst proportional mit dem Quadrate der Stromstärke. Beide Leiter äufsern endlich dieselbe magnetische und inductive Thätigkeit.

Schon vor lauger Zeit hat Faraday das Verhalten eines Körpers mitgetheilt, der beide Arten der Leitung zn besitzen scheint. Es ist das Schwefelsilber. In der vierten Reihe seiner Experimental-Untersuchungen über Elek(ricität ${ }^{1}$ ) findet sich die Stelle, in der er die sonderbaren Eigenschaften desselben beschreibt. Während sonst kein Punkt, der in jenen für den Galvanismus so wichtigen $\mathbf{A b}$ handlungen angeregt wird, unerörtert geblieben ist, hat, so viel mir bekannt, dieser Gegenstand bis jetzt keine weitere Beachtung gefunden. Ich gebe zuerst das Thatsächliche wortgetreu hier wieder, da ich mich in der Folge darauf zu beziehen habe.

\$. 432. "Die Steigerung der Leitungsfähigkeit mancher Substanzen, besonders für Elektricität von boher Spannung, durch die Wärme ist wohl bekannt. Kürzlich ist mir ein aufserordentlicher Fall dieser Art für Elektricität von schwacher Spannung oder die der Volta'schen Säule vorgekommen, die im directen Widerspruch steht mit dem Einflufs der Wärme auf metallische Körper, wie er von $\mathrm{Humphry}$ D a v y beschrieben worden ist. “

\$. 433. "Die Substanz, welche diese Erscheinung zeigt, ist das Schwefelsilber."

\$. 434. "Als ein $\frac{1}{2}$ Zoll dickes Stück desselben zwischen die mit den Polen einer Volta'schen Batterie von 20 Paaren 4 zöll. Platten verbundenen Platinspatel gebracht und ein Galvanometer mit in den Kreis eingeschaltet wurde, wich die Nadel ein wenig ab, als Anzeige einer schwachen Leitung. Als ich die Platinpole und das Schwefelsilber zusammenprefste, steigerte sich die Leitungsfähigkeit, so wie das Ganze warm wurde. Als ich unter das zwischen den 1) Pong. Ann. Bd. 31., S. 241. 
Polen befindliche Schwefelsilber eine Lampe stellte, nalim die Leitung rasch mit der Hitze zu, und zuletzt sprang die Nadel in eine feste Stellung über, indem das Schwefelsilber wie ein Metall leitete. Als nach Entfernung der Lampe die Wärme abnahm, kehrten sich die Erscheinungen um; die Nadel fing erst ein wenig zu vibriren an, verliefs dann allmälig ibre Querrichtung und nahm zuletzt sehr nahe die Stellung ein, welche sie ohne den Durchgang eines Stromes durch den Galvauometerdraht eingenommen haben würde."

\$. 435. "Zuweilen, wenn der Contact des Schwefelsilbers mit den Platinpolen gut, die Batterie frisch geladen, und die Temperatur aufangs nicht zu niedrig war, reichte der elektrische Strom der Batterie für sich hin, das Schwefelsilber in sciner Temperatur zu erhöben, und danu nahm diefs ohne Anwendung äufserer Wärme gleichzeitig auch an Leitungsfähigk eit zu, bis der erkaltende Einflufs der Luft die Wirkung bescbräukte. In solchen Fällen war es meistens nöthig, das Ganze eigends abzukühlen, um die umgekelurte Reihe von Erscheinungen zu erhalten. "

\$. 436. "Zuweilen nahmen auch die Wirkungen von selbst $a b$, und waren nicht eher zu erneuern, als bis das Schwefelsilber mit einer frischen Fläche auf den positiven Pol gelegt worden war. Diefs war die Folge besonderer Resultate einer Zersetzung, auf welche ich in der Abtheilung über elektro - chemische Zersetzung zurückkommen werde, und welche dadurch vermieden wurde, dafs ich die Enden zweier Platindrähte in die entgegengesetzten Enden ciner in einem Glasrohre geschmolzenen Portion Schwefelsilber steckte und dann diese Vorrichtung zwischen dic Pole einer Batterie brachte. "

\$. 437. "Das heifse Schwefelsilber leitete stark genug, un, wie ein Metall, helle Funken mit Kohle u. s. w. zu geben."

\$. 438. "Das natürliche Schwefelsilber und das Rothgülligerz zeigen dieselben Erscheinungen." "

\$. 439. "Es giebt meines Wissens aufser Schwefel- 
silber keinen andern Körper, welcher, so lange er heifs isl, linsichtlich seiner Leitungsfähigkeit für Elektricität von niederer Spannung mit den Metallen verglichen werden kann, und gauz unähnlich ihnen, diese Fähigkeit beim Erkalten verliert, während sie bei den Metalleu in Gegentheil zunimmt."

Ueber die in \$. 336. angedeutele Zersetzung findet sich in den folgenden Abhandlungen nur diese nähere Mit(heilung ') :

\$. 552. "Wenn Schwefelsäure durch die Säule zersetzt wird, scheidet sich der Schwefel am negativen Pole aus; wird aber Schwefelsilber auf änliche Weise zersetzt, so erscheint der Schwefel am positiven Pole. Und wenn in letztern Falle ein heifser Platiupol angewandt wird, um den abgeschiedenen Schwefel zu verflüchtigen, so ist die Bezichung dieses Poles zu dem Schwefel genau dieselbe, wie die Beziehung des nämlichen Poles bei dessen Eintauchung in Wasser zum Sauerstoff."

Meine erste Sorge war, obige Versuche unter solchen Umsländen zu wiederholen, welche eine Temperaturbestimmung gestatteten. Es ergab sich, dafs eine verhältnifsmäfsig geringe Erhöhung derselben, welche noch lange nicht die Gränzen unserer Thermometrie erreicht, jene bedeutende Aenderung des Leitungsvermögens herbeiführt. Bereits bei $180^{\circ} \mathrm{C}$. nimmt das Schwefelsilber den kleinen Widerstand an. Darin lag eine Aufforderung, diese Wirkung der Wärne genauer zu ennitteln.

Ich war so glücklich, unter den Schwefelmetallen ein zweites zu finden, das Halbschwefelkupfer (É), auf welches die Wärme ganz ähnlich einwirkt. In Folgenden werde ich es zuerst besprechen, da seine Eigenschaften uns zum Studium des Scbwefelsilbers nützlich seyn werden.

$$
\text { Das Halbschwefelkupfer (É). }
$$

Das Material zu meinen Versuchen bereitete ich durch Erhitzen von fein zertheiltem Kupfer und Schwefelblumen.

1) Fünfte Reihe in Pogg. Ann. Bd. 32, S. 448. 
Um das Metall in jenem Zustaude und rein zu erbalten, ward es durch Zink aus der angesäuerten Lösung seines schwefelsauren Salzes gefällt. Die Wasserstoffentwicklung, welche die freie Säure hervorruft, verhindert hierbei, dafs sich das Kupfer zusammenhängend ausscheidet. Man bekommt in kurzer Zeit den ganzen Gehalt der Flüssigkeit als eine schwammige Masse, die mit verdünnter Salzsäure gekocht, darauf ausgewaschen und getrocknet wurde. Bei der grofsen Zertheilung geht die Verbindung des Kupfers und Schwefels bereits bei wenig erhöhter Temperatur unter Erglühen von Statten. Die Masse, die sich bildet, lei. tet gut und besteht aus Halbschwefelkupfer und etwas Einfach-Schwefelkupfer. Dieselbe Neigung, welche das Oxydul, Chlorür, Bromür des Kupfers besitzen, noch ein Alom ihres elektro-negativen Bestaudtheiles aufzunebmen, äufsert auch das Halbschwefelkupfer, und zwar in hohem Grade.

So entstebt bekanutlich Einfach-Schwefelkupfer, wenn man gepulvertes Halbschwefelkupfer in der Kälte mit Salpetersäure übergiefst. Die Säure entzieht in niedriger Temperatur nur die Hälfte des Kupfers; die andere bleibt in Verbindung mit allem Scliwefel zurück.

Das Halbschwefelkupfer verbindet sich leicht mit geschmolzenem Schwefel und verwandelt sich in EinfachSchwefelkupfer. Man erhält letzteres mit derselben blauen Farbc, die der Kupferindigo besitzt, wenn man gepulvertes Halbschwefelkupfer mit Schwefelblumen erbitzt. Die Temperatur darf den Siedepunkt des Schwefels nicht mit übersteigen, indem schon vor der Glübhitze theilweise Zersetzung erfolgt. Die Analyse des künstlichen Kupferindigos ergab eine Zusaminensetzung, die der Formel Éu eutsprach; das Einfach-Schwefelkupfer zeigt sich, sowohl weun es auf diesem trocknen, als wenn es auf nassem Wege durch Fällung eines Kupferoxydsalzes mit Schwefelwasserstoffgas erhalten worden, als ein vortrefflicher metallischer Leiter der Elcktricilit. Eine Messung des Leitungsvermögens ist 
leider uumöglich, da es sich nicht in cohärentem Zustande darstellen lärst.

Das Halbschwefelkupfor ist dagegen bei gewöhnlicher Temperatur ein sehr schlechter Leiter. Diese Behauptung widerspricht allen Angaben meiner Vorgänger, welche dasselbe zu den an besten leitenden Schwefelmetallen zählen. Sie untersuchten jedoch eine Masse, welche eine Quantität Einfach-Schwefelkupfer enthielt. Letztere bedingt die gefuudene Leitungsähigkeit, wie das Folgende darthun wird.

In den Lehrbüchern d.r Chemic wird angegeben, das Einfach-Schwefelkupfer verlicre in der Glühbitze die Hälfte seines Schwefels. Dicse Angabe ist nicht ganz richtig. Ein hleiuer Theil Schwefel wird hier mit der gröfsten Hartnäckigkeit mehr festgehalten, als der Formel $\mathrm{Cu}$ entspricht. Derselbe entweicht noch nicht sogleich, wenn das Schwefelkupfer geschmolzen wird, was erst bei dem stärksten Rothglühen erfolgt; er kann sogar die Weifsglühhitze läıgere Zeit ertragen.

Es crgab sich dieses, als das Pulver, das durch Erhitzen von Kupfer und Schwefel bereitet war, im Hessischen Tiegel vor der Esse niedergeschmolzen wurde. Die Temperatur erreichte dabei gewöhnlich die Weifsglühhilze. Un der Masse eine regelmälsige Gestalt zu geben, wurde sie iin flüssigen Zustande in eine Form aus Gufseisen gegossen, welche ganz die Einrichtung derjenigen hatte, die zur Bereituug der Aetzkalistangen dient. Es hängt ganz von der Dauer der Schmelzung $a b$, welche Leitungsfähigkeit die erkalteten Cylinder besitzen sollen. Je läuger jene gewesen, desto gröfser ist ihr Widerstand, bis sie zuletzt fast so schlecht wie Schwefelsilber bei gewöhulicher Temperatur leiten. Das Schwefelkupfer, welches man bei möglichst kurzer Schmelzung erbält, besitzt eiten unkrystallinischen Bruch und eine etwas dunklere Farbe. Die Cylinder zeigen cive Menge unregelmäfsiger Absonderungssprünge, welche von der raschen Abkühlung herrühren und durch Anwärmen der eisernen Form vor dem Eingiefsen vermiudert werden können; sie fehlen ganz, wcun eine gröfsere Masse 
dicses Schwefelkupfers im Tiegel langsam erkaltet. Diese Cylinder sind sehr gute metallische Leiter. Ihr Widerstand ist natürlich verschieden, wenn sic aus verschiedenem Gusse herrubren. Bei dem besten Leiter, den ich gemessen., betrug er bei einem Cylinder, dessen Dicke $5,5^{\text {min }}$ und dessen Länge $47,5^{\mathrm{mm}}$ war, $30,6^{\mathrm{mm}}$ des Platindrabtes meines Rheostates; der Draht hatte einen Durchmesser von 0,4987mur. Die Leitungsfähigkeit war daher $\frac{1}{7 \delta}$ der des Platins. Un den Widerstand messen zu können, müssen die Enden der Cylinder in möglichst feste und iunige Berührung mit den metallischen Theilen der Kette gebracht werden. Diesen Zweck erfüllte mir folgende Klemmschraube, welche in Fig. 1. Taf. I. in natürlicher Gröfse dargestellt ist. Sie besteht aus einem Stablstücke $a$, das bis über die Hälfte zur Aufnahme des Cylinders ausgebohrt ist. Durch zwei Schnitte, dic sich kreuzen, ist die Hülse aufgeschlitzt und auf ihrer Oberfläche mit einer konisch zulaufenden Schraube versehen. Auf dieselbe pafst die Mutter $b$, welche zwei Löcher hat, so dafs sie sich durch den Schlüssel $c$ mit grofser Kraft aufschrauben läfst, wenn das Stück $a$ eingeklement ist. Die Hülse wird dadurch sehr fest gegen das Ende des Cylinders geprefst. Dasselbe wird vor dem Einstecken abgerundet, was am Bequemsten geschieht, indem man es einige Augenblicke durch eine Fraise auf der Drehbank laufen läfst. Die Berührung wird noch zuverlässiger, weun man das Ende mit einem dünnen Metallblättchen umwickelt. Das Stück $a$ ist an seinem anderen Ende zur Aufnahme des Verbindungsdrahts durchbobrt, den eine Druckschraube festhält. Nur bei dieser Einschaltung fand ich den Widerstand eines Cylinders proportional seiner Länge; bei weniger genauer Berührung bildet sich ein Uebergangswiderstand, der fortwährend sich ändert und den des Cylinders weit übertrifft. Die Messung geschah durch Hülfe der vortrefflichen Whe at stone-Kirchh offschen Differential - Vorrichtung.

Die Quantitit Schwefel, welche obiges Schwefelkupfer mehr enthält, als die Formel '́u verlangt, ist noch beder. 
tend genug, um von einer genauen Analyse dargethan zu werden. Die Cylinder, deren Widerstand ich angegeben, besafsen nämlich folgende Zusammensetzung:

$1,8322 \mathrm{Gr}$. enthielten $\left\{\begin{array}{l}\text { freier Schwefel }=0,1397 \\ \ldots\end{array}\right.$

0,3843 Gr. Schwefel $\{\dddot{S} \dot{B} a=1,774$ daher $S=0,2446$

oder in 100 Theilen:

$$
1,4456 \text { Gr. Kupfer }(\mathrm{Cu}=1,811)
$$

$$
\begin{aligned}
& \text { 20,97 Schwefel } \\
& \mathbf{7 8 , 9 0} \text { Kupfer } \\
& \text { 99,87. }
\end{aligned}
$$

Nach den Aequivalenten $(S=200,75)$ ist die Zusammensetzung des $\mathbf{E}$ :

$$
\begin{aligned}
& \text { 20,24 Schwefel } \\
& 79,76 \text { Kupfer. }
\end{aligned}
$$

Nehmen wir die Zusammensetzung unserer Masse in ruuden Zahlen:

$$
\begin{aligned}
& 21 \text { Schwefel } \\
& 79 \text { Kupfer, }
\end{aligned}
$$

so sehen wir, dals ungefähr 1 Proc. Schwefel mehr darin enthalten ist, als der Formel Ću entspricht. Denu die 79 Theile Kupfer verlangen 20,04 Schwefel, um $\mathfrak{C} u$ zu bilden. Es unterliegt keinem Zweifel, dafs dieser überschüssige Schwefel mit einem Theile des Halbschwefelkupfers zu Einfach-Schwefelkupfer vereinigt ist. Da er hierzu ungefähr 5 Theile bedarf, so würde unser Schwefelkupfer 6 Proc. '́u enthalten.

Noch auf einem andern Wege überzeugte ich wich von dem überschüssigen Schwefelgehalte. Derselbe entwich nämlich allmälig, als die gepulverte Masse in der Kugel einer Glasröhre in einem Strome getrockneten Kohlensäuregases über der Berzelius'schen Lampe geglüht wurde; er setzte sich theils als Blumen in den kältern Theilen der Röhre an, theils ward er voun Gasstrome fortgeführt. Bei Anwendung von 2,3715 Gr. zeigte die Röhre nach $1 \frac{1}{2}$ stündigem Rothglühen einen Gewichtsverlust von $0,0178 \mathrm{Gr}$, als der 
Anflug durch Erhitzen entfernt war. Es ist hierbei nölhig, dafs die Kohlensäure-Atmosphäre sich fortwährend crneuere. Wird der Strom unterbrochen, so hört auch das Entweichen des Schwefels auf. Trotz des langen Glübens war der überschüssige Schwefel noch nicht ganz ausgetrieben. Das Pulver leitele nach dem Erkalten noch gut. Das Austreiben wird zwar erschwert durch das Zusammenbacken des Pulvers; wir werden hiernach sogleich sehen, dafs dasselbe sich nicht vollständig bei dieser Temperatur wegschaffen läfst.

Dieses wird uur durch eine stärkere Hitze erreicht; die Masse mufs lange Zeit in geschmolzenen Zustande gehalten werden. In der Leitungsfähigkeit offenbart sich die Gegeuwart der kleinsten Quantität des Einfach-Schwefelkupfer; das Galvanometer ist hierauf das empfindlichste Reagens. Dasjenige Schwefelkupfer, welches den gröfsten Widerstand besitzt, müssen wir für das reinste erklärcn. Ich habe Cylinder gemessen, deren Widerstand bei einer Länge von $15,2^{\mathrm{mm}}$ und derselben Dicke von $5,5^{\mathrm{mm}}$ über 10,000 Meter Platiudraht bei der Temperatur $0^{\circ}$ betrug.

Beim Schinelzen in Tiegel wird das Entweichen des Schwefels durch die Ein wirkung des atmosphärischen Saucrstoffs unterstützt. Man bekommt daher auch leicht das schlechtleitende Schwefelkupfer, wenn man das gutleitende ohne Zusatz von Schwefel mehrmals umschmilzt. Umgekehrt läfst sich das schwachleitende durch Unschmelzen mit Schwefel in gutleitendes verwandeln.

Das Schwefelkupfer, dessen Widerstand ein grölserer ist, zeigt eine krystallinische Structur; die kleinen Krystallflächen treten deutlich auf dem Bruche hervor. Sehr häufig enthält die schlecbtleitende Masse eiuzelne Kupfertheilchen. Da sie das Leitungsvermögen nicht ändern und gesondert erscheinen, so ergiebt sich daraus, dafs das Schwefelkupfer nicht, wie die meisten anderen Schwefelmetalle, mit den Matallen zusammenschmilzt.

Ein sehr auffallendes Verhalten zeigt das schlechtleitende Schwefelkupfer in der Glühhitze. Als ich nämlich 
zar Controle einen Cylinder desselben in wohlgetrocknetem, reinem Kohlensäuregas über der Lampe glühte, wuchsen auf seiner Oberfläche aus einer Menge von Puncten böchst zierliche Kupferbaare hervor. Sie werden nicht selten 3-4"' lang, krüminen und gruppiren sich zu Locken; es entstehen die sonderbaren Bildungen, welche auch im Mineralreiche vorkommen und so sehr an die Producte der organischen Natur erimnern. Es entweicht hierbei kein Schwefel, kein Gewichtsverlust findet statt, dagegen zeigt der Cylinder nach dem Erkalten einen weit kleinern Widerstand. Demnach zersetzt sich ein Theil des Halbschwefelkupfers in freies Kupfer und Einfach-Schwefelkupfer. Merkwürdiger $W$ eise geschieht diefs in derselben Tempetur, iu der letzteres bereits zerfällt. Die Zerlegung findet uur an der Oberläche, nicht in Innern des Cyliuders statt; sie nimmt rasch ab, in dem Mafse, als sich die Menge des Cu vermehrt und wird nach einiger Zeit unmerklich. Daher zeigt sich auch die Erscheinung nicht an Cylindern, welche bereits einen kleinen Widerstand besilzen; sic lehrt aufserdem, dafs der Sclıwefelüberschufs bei gewöhnlicher Glühhitze nicht vollständig ausgetrieben werden kann.

In starker Glühhitze, in der das Halbschwefelkupfer schmilzt, tritt dic Zersetzung also wehr eiu. Hiervon überzeugte ich mich, indem ich schlechtleitendes Schwefelkupfer, das sich in einem Schiffchen aus hessischem Thone befand, in einem Flintenlaufe mehrere Stunden möglichst stark erbitzte, während Kohlensäure darüber strömte. Die Masse, welche geschmolzen gewesen war, zeigte nach dem Erkalten nur auf der Oberfläche einige wenige Kupferhaare, die also wäbrend der Abkühlung sich gebildet halten. Wollte man daher ein Stück Halbschwefelkupfer blofs durch Anwendung von Wärme vollständig zu Kupfer reduciren, so müfste man es abwechselnd einer schwachen und starken Glühhitze aussetzen. Das Kupfer, das sich in ersterer ausscheidet, wäre zu entfernen, ehe letztere angewendet wird, damit es sich nicht wieder mit dem danu austretenden Schwefel verbindet. 
Die freiwillige Zersetzung erschwert aufserordentlich die Bereitung eines vollkommen reinen Halbschwefelkupfers. Denn die in die Form gegossene Cylinder unterliegen derselben ebenfalls beim Erkalten; sie bedecken sich auf der Oberfläche mit einigen Kupferbaaren, während das einfache Schwefelkupfer sich einmengt und ihren Widerstand verringert. Nur einmal erhielt ich einen Gufs, der hiervon eine Ausnahme machte. Die Cylinder desselben hielten sich auch bei längerem Glühen unverändert und besafsen den grofsten Widerstand, den ich überhaupt gefunden und den ich eben mitgetheilt. Vergebens habe ich gesucht, sie wicderzuerbalten, dic Leitungsfähigkeit war stets wenigstens die zwei- oder dreifache, und alle Cylinder zersetzten sich theilweise in der Glühhitze.

In einer Temperalur, die unterhalb der Glühhitze liegt, tritt die Zerlegung nicht ein. Dahin deutete schon der Umstand, dafs die Kupferhaare sich vorzugsweise an der untern, heifsesten Seite des Cylinders bildeten. Ich versicherte mich hierüber auch direct und hielt einen schlechtleitenden Cylinder, der in einer mit Kohlensäure gefüllten Glasröbre eingeschmolzen war, mehre Stunden auf einer Temperatur über $300^{\circ}$. Er blieb unverändert. Hat aber in dieser Temperatur und schon bei $200^{\circ}$ die atmosphärische Luft Zutritt, erbitzt man ihn freiliegend in einem Luftbade, so besitzt er nach dem Erkalten einen kleinen Widerstand. Dabei hat sein Gewicht um einige Milligramm zugenommen; es ist Sauerstoff aufgenommen worden und dainit zugleich die Bildung von Einfach - Schwefelkupier eingetreten; der Procefs der Kupfervitriolerzeugung bat begonnen, den weiter hier zu verfolgen, uns abführen würde.

Bei nicderer Temperatur läfst sich nicht ein reives Halbschwefelkupfer bereiten; stets bildet sich mit demselben eine beträchtliche Menge Cu. Mau mag Kupfer und Schwefel noch so feingetheilt und innig gemengt, und noch so genau nach den Aequivalenten abgewogen erhitzen: stets ist die Verbindung ein gut leitendes Pulver. Auch auf nassein Wege bei der Behandlung von frisch gefältem Kupfer- 
chlorür mit Schwefelwasserstoffgas erhielt man kein anderes Resultat. Alles natürliche Halbschwefelkupfer, welches ich untersuchte, fand ich in Uebereinstimmung mit den frïheren Angaben gutleitend. Ich glaubte in Anfange meiner Untersuchung, dafs die Dimorphie den Unterschied des Leitungsvermögen bedinge, indem bekanntlich das natürliche und geschnolzene Halbschwefelkupfer ganz verschicdeue Krystallformen besitzen. Allein nach den obigen Erfahrungen zweifle ich nicht mehr, dafs auch die Leitungsfähigkeit des natürlichen Kupferglanzes von einem geringen Gebalt an $\dot{C} u$ herrührt. Man könnte uugekehrt jelzt die Dimorphie des Kupferglanzes bezweifeln, wenn nicht der Silberkupferglanz existirle.

Wenden wir uns nach diesen nothwendigen chemischen Erörterungen zu dem elektrischen Verhalten des Halbschwefelkupfers. Der grofse Widerstand, welchen dasselbe bei gewöhnlicher Temperatur besitzt, nimmt sehr rasch beim Erwärmen ab. Um ibn näher untersuchen zu können, wurde un den Cylinder, der in der oben beschricbenen Klemmschraube befestigt war, ein weites Glasrohr so ungeschmolzen, dafs es in zwei enge Röhrchen auslief. Während getrocknetes Kohlensïuregas durchströmte, konnten die Platindrähte, die zur Verbindung dienten, mit den Röhrchen zusammeugeschmolzen werden (Fig. 2 Taf. I.) Das Schwefelkupfer war auf diese Weise in der Almosphäre eines indifferenten Gases eingeschlossen und licfs sich bequem den verschiedenen Temperaturen eines Oelbades aussetzen. Letzteres bestand aus drei concentrischen in einander hängenden Kupfercylindern. In der Mitte des innersten Gefäfses wurde das Schwefelkupfer horizontal aufgehängt, indem die Drähte durch eine Oeffnung des Deckels hindurchgingen und durch einen Kork gehalten wurden. Zu jeder Seite der Röhre befand sich ein Thermometer sowie ein Metallblech, durch welches sich beim Auf- und Abbewegen eine gleichförnige Mengung und somit dicselbe Temperatur in der Flüssigkeit herstellen liefs. Dic 
Wärme lieferte eine Argand'sche Lampe mit constautem Niveau.

Das Halbschwefelkupfer leitet den galvanischen Stroin als Elektrolyt; es wird von demselben so zerlegt, dafs Kupfer am negativen, Schwefel ain positiven Ende des Cylinders sich ausscheidet. Ein Grove'sches Element genügt, um diese Wirkung deutlich hervorzurufen, wenn man dem Cylinder eine Temperatur über $110^{\circ}$. C. giebt. Das Kupfer tritt wieder meist in drahtförmigen Gestalten auf; es drängen sich aus dem Innern Büschel von Haaren hervor, die stärker, wie die eben durch freiwillige Zersetzung erhaltenen, sind. Sie erscheinen besonders zierlich, wenn man sie unter dem Mikroskope betrachtet. In Innern der Masse findet sich das Kupfer in der Form von Blällchen. Die Ausscheidung beginnt am äufsersten negativen Ende und rückt dem positiven zu. Der Cylinder berstet und zerklüftet an dieser Stelle und bricht nicht selten entzwei.

Will man das Wachsen der Kupferhaare beobachten, so ist natürlich das Oelbad zu vermeiden. In diesem Falle wäblt man einen stärkern Strom von $4-6$ Elementen, der durch die Wärmeentwicklung den Cylinder auf der nöthigen Temperatur erhält. Die Zersetzung läfst sich dann mit dem Auge verfolgen.

Der Schwefel tritt am positiven Ende nicht frei auf, sondern verbindet sich hier zu Einfach-Schwefelkupfer, das sich in der Masse verbreitet. Leitet man nur kurze Zeit den Strom eines Elementes durch den hiureichend erwärmten Cylinder, und untersucht darauf seinen Widerstand bei gewöhulicher Temperatur, so findet man letzteren bedeulend verringert.

Noch durch cine andere Erscheinung äufsert sich die Zersetzung: die ausgeschiedenen Bestandtheile rufen näınlich Gegenströme bervor, Kupfer, Halbschwefelkupfer und Einfach-Schwefelkupfer bilden eine wirksame Kelle, in der ersteres das positive, letzteres das negative Metall abgiebt. Durch die Wippe läfst sich leicht dic Polarisation darthun. Man leitet den Strom eines Grove'schen Elemen- 
tes in erhöhter Temperatur durch den Cylinder und verbindet seine Euden darauf sogleich mit den Drähten eines Galvanometers. Die Gegenströme sind nicht thermoelektrischer Natur, da ibre Intensität eine zu bedeutende ist. Genau messen läfst sich letztere nicht, da sie veränderlich ist und abuinmt, wie die Menge des Einfach-Schwefelkupfers zuniumt. Sie erreicht bereits bei dem Strome cines Grove'schen Elementes ihr Maximun, wenn Cylinder von mäfsiger Länge gewählt sind und ihr Widerstand durch Temperaturerhöhung binreichend vermindert ist. Bei einem Versuche mit einem frischen Cylinder fand ich durch die Poggendorff'sche Compensationsmethode eine elektromotorische Kraft, welche etwa 'To von einer Daniellschen Kette betrug.

Die Zersetzung nimmt rasch bei der Fortdauer des Stromes ab. In dem Maafse nämlich, als das Einfach-Schwefelkupfer sich vermehrt, wird der Cylinder ein immer besserer metallischer Leiter. Läfst man den Strom eines Grove'schen Elementes mehre Stunden ununterbrochen in erhöhter Temperatur bindurchgehen, so zeigt sich gewöhnlich zuletzt weder ein Gegenstrom, noch eine Zersetzung wehr. Das von Kupfer freie Ende des Cylinders ist cin vortrefflicher metallischer Leiter geworden und wird nicht mehr in erhöbter Temperatur zerlegt. So verhalten sich von vorn herein die bestleitenden Sorten Schwefclkupfer, die bei möglichst kurzer Schmelzung erbaltenen Glieder. Sie zeigen keine der eben besprochenen Erscheinungen. Je schlechter das Material leitet, desto geeigneter ist es hierzu.

Die rasche Abnahme, welche der Widerstand des Halbschwefelkupfers beim Erwärmen erleidet, erklärt sich einfach dadurch, dafs ein Erweichen, eine Beweglichkeit der Theilchen eintrit. Dic Cylinder sind bei gewöhnlicher Temperatur so spröde, dafs sie sogleich durchbrechen, wenn man sie zu biegen versucht. In höherer Temperalur läfst sich dieses leicht bewerkstelligen. Ich halte das ganz reine Halbschwefelkupfer nur für einen elek troly tischeu Leiter; wenigstens kommt die bedeutende Zunahme seines Leitungsver- 
mögens nur auf Rechnung seiner elektrolytischen Natur. Es besitzt entweder gar keine, oder ganz geringe metallische Leitung, wie sehr man sie auch nach den oplischen Eigenschaften vermuthen sollte. Es liefse sich dieses strenge beweisen, wenn man die Bildung des Ću bei der Elektrolyse verhindern könnte. Man brauchte blofs die Menge des ausgeschiedenen Kupfers mit den Zersetzungsproducten eines eingeschalteten Voltameters zu vergleichen. Durch jenes secundäre Product wird jedoch bald der grö́sere Theil des Stroms metallisch geleitet und so nur einc verhältnifsmälsig geringe Quantität Kupfer ausgeschieden. Die Frage liefse sich aufserdem noch durch das in der Einleitung hervorgeliobene, von Poggendorff angegebene Verfahren mit Bestimmtheit entscheiden. Es" wäre zu untersuchen, ob der Widerstand eines geraden metallischen Lciters in den verschiedenen Temperaturen ein anderer werde, weun er als Axe eines Cylinders von Halbschwefelkupfer dient. Der Versuch bietet jedoch zu grofse praktische Schwierigkeiten und respective von vorn herein kein Resultat. Denn, wie wir sogleich sehen werden, bleibt der Widerstand des Halbschwefelkupfers auch in den höheren Temperaturen immer ein beträchtlicher, verglichen mit den Widerständen der metallischen Leiter, die wir hicr anwenden könnten.

Die Bestimmung des Widerstandes, welchen unser Schwefelmetall bei den verschicdenen Temperaturen annimmt, bietet grofse Schwierigkeit, wenn sie genau seyn soll; keine der bekannten Methoden genügt. Das Verfahren, welches man gewöhnlich bei Elektrolyten, in denen Gegenströme auftreten, anwendet, wonach man den Widerstand zweier verschiedenen Längen mit derselben Stromstärke mifst, ist in unserem Falle nicht brauchbar. Das längere Durchleiten eines stärkeren Stromes ändert zu sehr die chemische Natur des Materials. Ich habe vorgezogen, einstweilen den Widerstand mit möglichst schwachen und momentanen Strömen durch die Differential - Vorrichtung zu messen und die 
Polarisation zu vernachlässigen. Der Strom eines Grove'schen Bechers wurde durch Einschaltung eines Neusilber-Rheostats bedeutend geschwächt und immer nur für so kurze Zeit geschlossen, als nöthig war, um die astatische Nadel des Galranometers nach der einen oder anderen Seite abzulenken. Die folgenden Resultate können daher nur angenähert richtig seyn. Ich theile sie mit, weil sie eine Folgerung gestatten, die durch das Experiment modificirt werden kann.

Ein Cylinder, der aus dem am schlechtesten leitenden Gusse, den ich erhalten habe, gewählt war, und einc Länge von $15,2^{\mathrm{mm}}$, eine Dicke vou $5,5^{\mathrm{mm}}$ besals, zeigte folgende Widerstände :

Bei der Temp. $0^{\circ}$ C. 9.1130 Meter Platindraht, dessen Durchmesser 0,4987 $\mathrm{mm}$.

$\begin{array}{lrrrc}" & 10 & 9 . & 681 & \text { Meter } \\ " & 51 & 9 . & 120 & " \\ " & 67 & 9 . & 65 & " \\ " & 85 & 9 . & 40 & " \\ " & 103 & 9 . & 22,4 & " \\ " & 107 & 9 . & 9,4 & " \\ " & 113 & 9 . & 9,3 & " \\ " & 136 & 9 . & 5,2 & " \\ " & 152 & 9 . & 3,8 & " \\ " & 184 & 9 . & 2,2 & " \\ " & 192 & 9 . & 2 & "\end{array}$

Wir sehen in dieser Reihe den Widerstand innerhalb des kleinen Temperaturintervalls zwischen $103^{\circ}$ und $107^{\circ}$ um beinahe das $1_{\frac{1}{2}}$ fache abnehmen, von 201,6 Meter auf 84,6 fallen. Eine so grofse Verringerung bewirkt sonst erst eine Temperaturerhöhung über $20^{\circ}$. Rührt die $\mathrm{Zu}$ nahme des Leituugsvermögens vom Erweichen der Masse her, so wird in dieser Temperatur dasselbe in slïrkerem Grade eintreten müssell. Dazu ist aber Wärme nöthig, welche demuach hier in gröfserer Menge aufgenommen werden mufs. Wir finden diesen Schlufs bestätigt, wenn wir erwärntes Halbschwefelkupfer erkalten lassen und die Zeiten, in denen sich die Temperatur un bestimmte Inter- 
valle erniedrigt, beobachten. Ich wählte eineu Cylinder, dessen Durchmesser und Höhe $26^{\mathrm{mm}}$ betrugen, und schlols ihn in eine dünne Hülle aus Messing. In seiner Mitte befand sich eine cylindrische Oeffnung, in welche der Behälter eines Therınometers genau pafste. Er wurde selbst in eine Oeffnung des Deckels der Hülle durch einen Kork gebalten. Nachdem der Cylinder im Luftbade über $20^{\circ}$ erwärmt worden, liefs ich ihn in freier Luft erkalten. Das Thermometer gebrauchte folgende Zeiten, um dic beistehenden Temperaturintervalle zu durchfallen:

\begin{tabular}{|c|c|c|c|}
\hline \multicolumn{4}{|c|}{ No. I. } \\
\hline $\begin{array}{l}\text { 'Temperatur. } \\
250^{\circ} \mathrm{C} \text {. }\end{array}$ & Zeit. & $\begin{array}{l}\text { Temperatur. } \\
103^{\circ} \mathrm{C}\end{array}$ & Zeit. \\
\hline 240 & $45^{\prime \prime}$ & 102 & $20^{\prime \prime}$ \\
\hline 230 & 47 & 101 & 29 \\
\hline 220 & 48 & 100 & 38 \\
\hline 210 & 52 & 99 & $4^{\prime} 35$ \\
\hline 200 & 51 & 98 & 120 \\
\hline 190 & 58 & 95 & 142 \\
\hline 180 & ' 3 & 90 & 158 \\
\hline 170 & 17 & so & 240 \\
\hline 160 & 112 & 70 & 245 \\
\hline 150 & 120 & 60 & 330 \\
\hline 140 & 125 & 50 & 425 \\
\hline 130 & 138 & & \\
\hline 120 & 147 & & \\
\hline 110 & 2 & & \\
\hline 105 & 112 & & \\
\hline 104 & 15 & & \\
\hline 103 & 21. & & \\
\hline
\end{tabular}

Wir finden die Verzögerung im Sinken des Thermometcrs bei einer etwas niedrigeren Temperatur, als sie nach der Abnahme des Widerstandes zu erwarten war. Diefs werden vorzüglich zwei Umstände bewirken. Der Cylinder, den ich zu deın Erkaltungsversuch benutzte, rührt nicht aus demselben Gusse, wie derjenige, dessen Wider- 
stand ich bestimmte. Er wird etwas mebr Einfach-Schwefelkupfer enthalten, da er auf der Oberfläche sich mit Kupferhaaren beim Erstarren bedeckt hatte. Je gröfser aber die Menge des 'Cu ist, bei desto niedrigerer Temperatur tritt der Stillstand des Thermometers ein. Ich fand ihn bei einem zweiten Cylinder, der noch ans schlecht leitender Masse bestand, bei $97^{\circ} \mathrm{C}$., bei einem dritten, der zu den gutleitenden gehörte, aber noch die Elektrolyse zeigte, bei $79^{\circ}$. Bei einem vierten endlich, der zu den besten Leitern gehörte, trat keine auffallende Verzögerung mehr ein.

Folgende Tabellen lehren diefs für die beiden letzten Cylinder :

No. II.

No. III.

\begin{tabular}{|c|c|c|c|}
\hline $\begin{array}{l}\text { Temperatur. } \\
200^{\circ} \mathrm{C} .\end{array}$ & Zeit. & $\begin{array}{r}\text { Temperatur. } \\
200^{\circ} \mathrm{C} .\end{array}$ & Zeit. \\
\hline 190 & $56^{\prime \prime}$ & $190^{\circ}$ & $49^{\prime}$ \\
\hline 180 & $\mathbf{1}^{\prime}$ & 180 & 55 \\
\hline 170 & 18 & 170 & 60 \\
\hline 160 & 120 & 160 & l' 15 \\
\hline 150 & 136 & 150 & 144 \\
\hline 140 & 139 & 140 & 141 \\
\hline 130 & 150 & 130 & 149 \\
\hline 120 & 156 & 120 & 158 \\
\hline 110 & 213 & 110 & 27 \\
\hline 100 & 229 & 100 & 225 \\
\hline 90 & 249 & 90 & 240 \\
\hline 85 & 141 & 80 & 320 \\
\hline 84 & 24 & 70 & 423 \\
\hline 83 & 28 & 60 & 430 \\
\hline 82 & 30 & 50 & 525 \\
\hline 81 & 50 & & \\
\hline 80 & 110 & & \\
\hline 78 & 5 & & \\
\hline 70 & 230 & & \\
\hline 60 & 345 & & \\
\hline 50 & 55. & & \\
\hline
\end{tabular}


Die Hauptursache der mangelhaften Uebereinstimmung liegt sonach in der Methode selbst, durch welche wir dic latente Wärme nachweisen. Wir wissen nïmlich aus vielen Beispielen, dafs die Körper beim Erkalten ibre Schmelzwärmc weit unter der Temperatur, bei der die Aufnahme erfolgt, festhalten können, dafs die meisten sie nicht genau bei derselben Temperatur beim Erkalten angebon. Ebenso wird es sich hier verhalten und wir werden uns mit der Aunäberung begnügen müssen.

\section{Das schwefelsilber.}

Bei der Bereitung des Schwefelsilbers zu dem Zwecke, den wir hier verfolgen, muls man mit der gröfsten Sorgfalt die kleinste Quantitit freien Silbers auszuschliefsen suchen. Denn dasselbe schmilzt mit der Schwefelverbindung zusammen und verändert ihr Leitungsvermögen gänzlich. Vor Allem ist der Zutritt der Luft beim Schmelzen zu verhindern, da sie sogleich reducirend durch den Saucrstoff wirkt. Auf folgende Weise habe ich meine Cylinder dargestellt. Ausgewaschenes Chlorsilber wurde noch feucht in das Thongefäls eines Grove'schen Bechers gebracht und letzterer zusammengestellt, und geschlossen, wie gewöhnlich, nur dafs die Salpelersäure durch verdünnte Salzsäure crselzt war. Nach einigen Stunden halle der Strom das Chlorsilber reducirt. Ich mengte das feinzertheilte Mctall, als es ausgewaschen und getrocknet war, mit den erforderlichen Schwefelblumen und führte durch mäfsiges Erwärmen im Porcellantiegel die Verbindung herbei. Das Schwefelsilber versetzle ich wicderum mit Schwefel und füllte es in Röhren ans schwerflüssigem Glase, die an dem cinen Ende zugeschmolzen waren. Nachdem das andere Ende in eine feine Spitze, die geöffuet blieb, ausgezogen war, wurden die Röhren in ein Gefäfs mit Saud bis zur Spitze eingesenkt und das Ganze in den Ofen gebracht. So lange der Schwefel aus der freien Oeffnung beim Erhilzen entwich, blieb der Ofen ungedeckt. Näherte sich die Schwefelflamme Jem Erlöschen, so wurde der Iom auf- 
gesetzt, das Feuer verstärkt und eine bestimmte Zeit unterhalten. Das Schwefelsilber schmitzt bei heller Glühbitze, einer Temperatur, bei der auch das Glas zu erweichen beginnt. Man muls durch einige Versuche die Zeit ermitteln wo ersteres eingetreten, ohne dafs die Hülle gelitten hat. Sie betrug für meinen Ofen 5 bis 6 Minuten. Die Spitze schmilzt gewöhnlich zu und ist im Innern noch mit einem Schwefeltropfen bckleidet. Das Schwefelsilber findet sich im unteren 'Theile der Röhre als ein Cylinder, dem sich leicht auf der Drehbank eine regelnäfsige Form geben läist.

Das Schwefelsilber bildet einc geschmeidige, etwas biegsame Masse, die sich leicht mit dem Messer schneiden läfst. Es besitzt cine schwarzgraue Farbe und beträchtlichen Metallglanz. Schon bei gewöhnlicher Temperatur beobachtet man die Zersetzung beim Durchleiten cines Stromes. Er cntsteht am negativen Ende, an der Stelle, wo das verbindende Metall anliegt, ein schwacher, weifser Silberfleck, der jedoch wenig zunimmt, da der Schwefel als Isolator bald den Strom gänzlich hemmt. Befestigt man die Enden des Cylinders, mit Platin-, oder Silberblech umhüllt, in den Klemmschrauben, die ich oben beschrieben, und erwärmt denselben im Oelbade, so treten schon bei einem Grove'schen Elemente die Erscheinungen ciu, wie sie Faraday mittheilt. Der Widerstand nimmt rasch ab. Ist ein Galvanometer mit einfacher Nadel eingeschaltet, so wird letztere immer melr abgelenkt und nimmt bei $180^{\circ} \mathrm{C}$. beinahe die Stelle ein, auf welche sie der Strom obne das Schwefelsilber bringt. Beim Erkalten geht sie wieder allmälig zurück. Leitet man auch lïngere Zeit den Strom in erhöhter Temperatur hindurch, so findet man dennoch nur eine ganz geringe Quantität Silber ausgeschicden. Da derselbe in einem Voltaneter eine beträchtliche Zersetzung bewirkt hätte, so muls er fast ganz metailisch geleitet worden seyn. Zwei verschiedeue Vorgänge können diefs herbeiführen: Das Schwefelsilber wird entweder ein metallischer Leiter, oder es bildet sich eine Ver- 
bindung von Silber zwischen den beiden Euden des Cylinders, die in der noch festen Masse sehr leicht zu Staude kommen mufs. Erstere Erklärung, die F a raday annimmt, scheiut durch deu Umstand nothwendig, dafs beim Erkalten der Widerstand wieder abuimmt. Wäre ein Silberfaden vorhanden, so sollte man glauben, derselbe inüfste bleiben und auch bei niederer Teuperatur eine gute Leitung herbeifuhren. Ich theilte ebenfalls in Anfange diese Ansicht und unternahm deshalb die genauere Untersuchung. Folgende Erfahrungen sprechen jedoch entschieden für die zweite Auffassung.

Ich umgab die beiden Enden eines Cylinders von etwa $20^{\text {mm }}$ Länge mit Zinkblech, einem Metalle, mit dem sich der Schwefel nicht verbindet und klemmte ihn damit ein. Darauf wurde er in die indifferente Atmosphäre von Kohlensäure eingeschmolzen, im Oelbade auf $190^{\circ}$ erbitzt, und nun erst der Strom eines Elementes durchgeleitet. Die einfache Nadel des Galvanometers wich kaum $5^{\circ}$ ab und kehrte bald auf $0^{\circ}$ zurück. Dasselbe trat ein, als der Stron verstärkt wurde, als zwei Elemente genominen wurden. Demnach leitet das Schwefelsilber auch bier noch als Elektrolyt; der frei gewordene Schwefel hemut den Durchgang der Elektricität. Wurde jetzt die Richtung des Stromes ungekehrt, so schlug die Nadel sogleich nach entgegengesetzter Seite gegen die Hemmung, oscillirte um $70^{\prime \prime}$ und schwankte unregelınäfsig zwischen $60^{\circ}$ und $70^{\circ}$; sie ging nicht zurück, so lange die Temperatur unterhalten wurde. Ueber eine halbe Stunde dauerte der Strom; er reducirte wäbrend dieser Zeit 0,172 Gr. Silber aus der Lösung des salpetersauren Salzes. Erst beim Erkalten kehrte die Nadel zurück, bereits unter $160^{\circ}$ kam sie auf $0^{0}$. Das Schwefelsilber zeigte nur eine Spur freies Silber au den Euden.

Dieser Versuch läfst sich nur durch die zsweite Erklärung deuten. Wälrend der kurzen Zeit, in welcher dic zuerst abyeschiedenen Bestandtheile dem eutgegengesetzten Strome den Durchgang gestatten, bildet derselbe sich eine 
metailische Verbindung durch ausgeschiedenes Silber, zwischen deu beiden Enden. Sic mufs aufserordentlich dünn seyn, da sie trotz der Kürze des Cylinders die Nadel nur 60 bis $70^{\circ}$ ablenkt, eine Ablenkung, der ein Widerstand von 50 bis 20 Meter meines Platindrahtes entspricht. Es darf uns daher nicht wundern, wenn dieser feine Faden oder diese Haut beim Erkalten, wo die Masse sich zusammenzicht, zerstört wird, zumal, da unterhalb $180^{\circ}$ wie wir bald finden werden, eine bedeutende Verfestigung des Schwefelsilbers eintritt.

Combinirt mau das Schwefelsilber am positiven Ende mit Halbschwefelkupfer, so verbindet sich der bei der Elektrolyse austretende Schwefel zu Einfach-Schwefelkupfer. Er verursacht keinen Widerstand mehr, sondern ruft starke Gegenströme hervor. In eine der beiden Schrauben, wurde ein Stück eines bestlcitenden Cylinders Schwefelkupfer eingesteckt und so ausgebobrt, dafs eine Hülse übrig blieb, die das Schwefelsilber umfafste. Iu erhöhter Temperatur, besonders oberhalb $180^{\circ}$, erregt der Strom eines Grove'schen Elements Gegenströme, welche an Inteusität die beim Halbschwefelkupfer erhaltenen noch übertreffen. Ihre elektromotorische Kraft schätze ich auf $\frac{1}{6}$ bis $\frac{1}{\dagger}$ derjenigen einer Daniell'schen Kette. Die wirksamen Theile, die die Polarisation bedingen, sind in diesem Falle Silber, Schwefelsilber, Einfach -Schwefelkupfer. Das Schwefelkupfer wird nicht zersetzt; davon hatte ich mich vorber überzeugt.

Nach längerem Durcbleiten unter diesen Umständen findet man das Silber in etwas gröfserer Menge; es bildet auch, wie das Kupfer, einzelne Haare, in der Nähe des negativen Poles. Doch wird immer nur ein kleiner Theil des Stromes elektrolytisch geleitet, da eine metallische Verbindung zwischen den Enden entsteht. Unterbält man den Strom bei dieser Combination während des Erkaltens, so geht die Nadel leise zurück. Der Widerstaud des Cylinders bleibt auch bei gewöbnlicher Temperatur ein geringer. In einem frischen Stücke, das auf obige Weise verbunden ist, erfolgt eine allmälige Zunahme der Leitung, wenn bei 
ederer Tumperatur der Strom unterhalten wird; die Na1 weicht immer mehr ab, natürlich um so langsamer, je eniger Erwärnnung stattgefunden. Es erklärt sich diefs cht. Der austretende Schwefel bewirkt hier dem Strome in Hemmuifs, das Einfach-Schwefelkupfer verbreitet sich Halbschwefelkupfer, die Elektrolyse kann fortdauern id den Silberfaden herstellen. Erbilzt man einen Cylin$r$, der auf diese Weise gutleitend geworden, über $180^{\circ}$ ist er es in der Regel nach dem Erkalten nicht mehr; $e$ metallische Verbindung ist zerstört.

Ich habe nur das Schwefelkupfer geeignet gefunden, n Widerstand an positiven Pole ganz zu verhindern. e Metalle vermögen es nicht. Sogar das Silber, von in man es sicher erwarten sollte, scheint sich nicht sozich mit dem austretenden Schwefel za verbinden. Wejstens findet man den Widerstand eines Cylinders, der t Silberblech eingeklemmt ist, in der Differential-Vorhtung, bei derselben Temperatur weit gröfscr, weun eil irkerer, wie wenn ein schwächerer Strom zum Messer nutzt wird. Erst in höberen Temperaturen hört der Un'schied auf.

Un annähernde Werthe der Widerstände des Schwesilbers für die verschiedenen Temperaturen zu erhalten, eb daher nur die Combination mit bestleitendem Schwekupfer tubrig, obgleich sie durch die Gegenströme ebenIs vieles zu wünschen läfst. Es war nöthig, beide Ena des Schwefelsilbers mit Schwefelkupfer zu verbinden, il bei einseitiger Herstellung ein schwacher Strom in un Systeme sich einstellt, der besonders in höhere Tempeuren sehr störend wird. Der Strom, den ein Grove'sches sment zum Messer lieferte, wurde wieder bedeutend geıwächt. Ein Cylinder, dessen Länge $\mathbf{2 0}^{\mathrm{mm}}$, dessen Durchsser $5,3^{\text {max }}$ betrug, hatte folgende Widerstände bei deu istebenden Temperaluren: 
Temperatur. Viderstand.

$81,1^{\circ}$ C. 9. 537 Meter Platindraht (Durchm. 0,4987min

93

113,2

9. 395

129,2

9. 142

148

9. 120

$15 \mathrm{~S}, 2$

9. 67

165,2

9. 40,5

170

9. 25,6

180,5

9. 13,8 "

195

9. 0,88 ”

9. 0,77 ”

Für niedrigere Temperaturen als $84^{\circ}$, habe ich keine Bestimmungen gemacht, da meine Widerstandsrollen nicht ausreichen.

Sämmlliche Erscheinungen, welche wir beim Schwefelsilber gefunden, sprechen für die elektrolytische Natur desselben. Die Abnahme des Widerstandes beim Erwärmen wird durch eine Erweichung bedingt. Dieselbe ist über $1 S 0^{\circ}$ C. bereits so beträchllich, dafs ein Stück unseres Schwefelmetalls, welches man längere Zeit auf einer solchen Temperatur in einem indifferenten Medium erhäl, seine Gestalt ändert und auf seiner unteren Seite mehr oder weniger die Form des Gefälses annimmt. Seine Oberfläche bedeckt sich mit feinen, glatten, glänzenden Krystallen, die Rhombendodekaëder sind, und nicht selten die Gröfse einiger Linien erreichen. Die Masse wird daher so weich, dafs die Theilchen sich zu Krystallen gruppiren könneu.

In der Reibe der Widerstände fällt sogleich die bedeutende Abuabme oberhalb $170^{\circ}$ auf. Hier wird daber eine starke Erweichung eintreten, demnach Wärme in gröfserer Menge aufgenommen werden müssen. Wir haben, um uns davon zu überzeugen, wie beim Halbschwefelkupfer zu verfahren. Ein Cylinder Schwefelsilber, der dieselben Dimensionen hatte uod in die Messinghülse pafste, gebrauchte zum Erkalten folgende Zeiten: 


$\begin{array}{lccr}\text { Temperatur. } & \text { Zeit. } & \begin{array}{r}\text { Tervperatur. } \\ 150^{\circ} \text { C. }\end{array} & \text { Zcit. } \\ 250^{\circ} \text { C. } & & 140 & 40^{\prime \prime} \\ 240 & 32^{\prime \prime} & 130 & 50 \\ 230 & 33 & 120 & 1^{\prime} 8 \\ 220 & 35 & 110 & 119 \\ 210 & 37 & 100 & 133 \\ 200 & 40 & 90 & 145 \\ 190 & 43 & 80 & 210 \\ 180 & 52 & & \\ 170 & 1^{\prime} 10 & & \\ 165 & 1 & & \\ 160 & 3 & & \\ 155 & 50 & & \\ 150 & 20 . & \end{array}$

Das Sinken des Thermometers verzögerte sich aın stärksten zwischen $164^{\circ}$ und 162". Bei einem anderen Cylinder trat der Stillstand bei $167^{\circ}$ ein. Die mangelhafte Uebereinstimmung der beiden Reihen wird auch hier durch die Methode selbst bedingt seyn.

Ueberblicken wir die Resultate der Untersuchung. Das Verhalten unserer beiden Schwefelmetalle bietet in elektrischer Hinsicht nichts Abnormes mehr. Ungeachtet die optischen Eigenschaften metallische Leiter in ihnen erwarten lassen, erweisen sie sich als Elektrolyte. Bereits weit unter ihrem Schmelzpmnkte, der erst bei heller Glühbitze eintritt, erweichen sie und nehmen latente Wärme auf. Es geschieht diefs nicht regelmäfsig, sondern das Halbschwefelkupfer bindel oberhalb $103^{\circ} \mathrm{C}$., das Schwefelsilber oberhalb $170^{\circ} \mathrm{C}$. eine gröfsere Menge. In dem Maalse, als sie erweichen, wird die Elektrolyse erleichtert, nimint ihr Widerstand ab.

In chemischer Hinsicht bietet das Resultat noch dadurch Interesse, dafs es gerade die beiden Schwefclinetalle sind, welche auch bier die gröfste Aehnlichkeit besitzen, welche sich in ihren Verbindungen vollständig vertreten, isomorphe Körper bilden.

Die meisten Schwefelverbiudungen der schweren Me- 
talle verhalten sich zum elektrischen Strome wie die Metalle selbst. Ibr Widerstand ist klein und nimmt beim Erwärnen zu. Ich habe nur die gewöhnlichen Metalle berücksichtigt. Es gehören in diese Klasse: Schwefelblei, Schwefelwismutb, Schwefelquecksilber ( $\dot{H}_{\mathrm{H} g}$ und $\dot{H}_{\mathrm{g}}$ ), Schwefelkupfer (Ć $\mathbf{C}$ ), so wie die Kiese: Schwefeleisen, Schwefelnickel, Schwefelkobalt.

In Allgemeinen gestattet die Farbe, mit welcher Schwefelwasserstoff die Verbindungen aus den Auflösungen der Salze niederscblägt, einen Scblufs auf ihr elektrisches Leitvermögen. Die genannten Schwefelmetalle werden alle schwarz gefällt. Während der rothe Zinnober isolirt, ist das schwarze Quecksilbersulfid, wie schon Muuck af Rosenschöld fand, cin vortrefflicher metallischer Leiter. $D a$ es nur pulverförmig $x u$ erbalten ist, so läfst sich sein Widerstand leider nicht messen.

Die Schwefelmetalle, welche mit einer anderen Farbe gefällt werden, isoliren den galvanischen Strow. Ich nenne Schwefelzink, Schwefelkadmium, Schwefelmangan, Schwefelantimon. Die drei letzten werden in hoher Temperatur bessere Leiter, bieten jedoch der genaueren Untersuchung grofse Schwierigkeiten. Schwefelkadmium ist nicht schmelzbar, sondern verflüchtigt sich in der Weifsglühhitze; Schwefelmangan vermochte ich vor der Esse ebenfalls nicht zum Flusse zu bringen. Erst bei der Glühhitze nimut der Widerstand der beiden Körper bedeutend ab. Beim Scliwefelantimon tritt die bessere Leitung uicht weit unterhalb des Schmelzpunktes ein. Es ist wahrscheinlich, dars in allen drei Fällen eine Elektrolyse eintritt, welche durch eine Er. reichung bedingt wird, jedoch vermag ich es nicht zu beweisen. Auf das Schwefelantimon werde ich vielleicht bei einer anderen Gelegenheit zurückkommen.

Aufser dem Schwefelsilber und Halbschwefelkupfer bildel noch das Zinnsulfür eine Ausnabme von obiger Regel. Sein Widerstand ist trotz der schwarzeu Farbe bei gewöhulicber Temperatur sehr grofs, nimmt aber beim Erwämen ab. Ich habe es nicht genauer sludiren können, da 
es seines weichen krystallinisch-blättrigen Zustandes wegen keine Einklemmung gestattet, und erst oberhalb $300^{\circ}$ dic bessere Leitung sich einstellt. Zinusulfid ist bekanntlich ein metallischer Leiter.

Ich war neugierig, das elektrische Verbalten der Verbindungen, welche Silber und Kupfer mit dem Selen eingehen, kennen zu lernen. Beide sind gute metallische Leiter. Halbselenkupfer wurde mehrmals ungeschmolzen, Sclensilber sowohl als Niederschlag, durch Selenwasserstoff gefällt, wie geschmolzen untersucht. Es folgen zum Schlufs ilure Widerstände, wobei der des Platins zur Einheit gewillt wurde:

\begin{tabular}{|c|c|c|}
\hline \multicolumn{3}{|c|}{ Selensilber. } \\
\hline Widerstand & bei $\quad 0^{0}$ & 24,5 \\
\hline " & bei 100 & 31,8 \\
\hline \multicolumn{3}{|c|}{ Halbselenkupfur. } \\
\hline Widerstand & bei $0^{\circ}$ & 155,5 . \\
\hline$"$ & bei 100 & 278,9 . \\
\hline
\end{tabular}

II. Veber die Construction eines Elektromelers; von Hankel.

(Migetheilt vom Irn. Verf. aus d. Ferhandlungen d. K. Sächs. Gesell. d. Viss. 1851 No. II.)

Die Verbesserung und Vervollkominnung der zur Wahrnehmung und Messung der Elektricität dienenden Instrumente ist für die Entwickelung der Elektricitätslehre von grofser Wichtigkeit, und es dürfte daher die Mitheilung der Construction cines solchen Instrumentes, welches alle bisher gebrauchten rücksichtlich der Empfindlichkeit bei Weitem übertrifft und doch zugleich eine Messung der Stärke der Elektricität, so wie die Erkennung ihrer positiven oder negativen Beschaffenheit gestattet, nicht überflüssig erscheincn. 


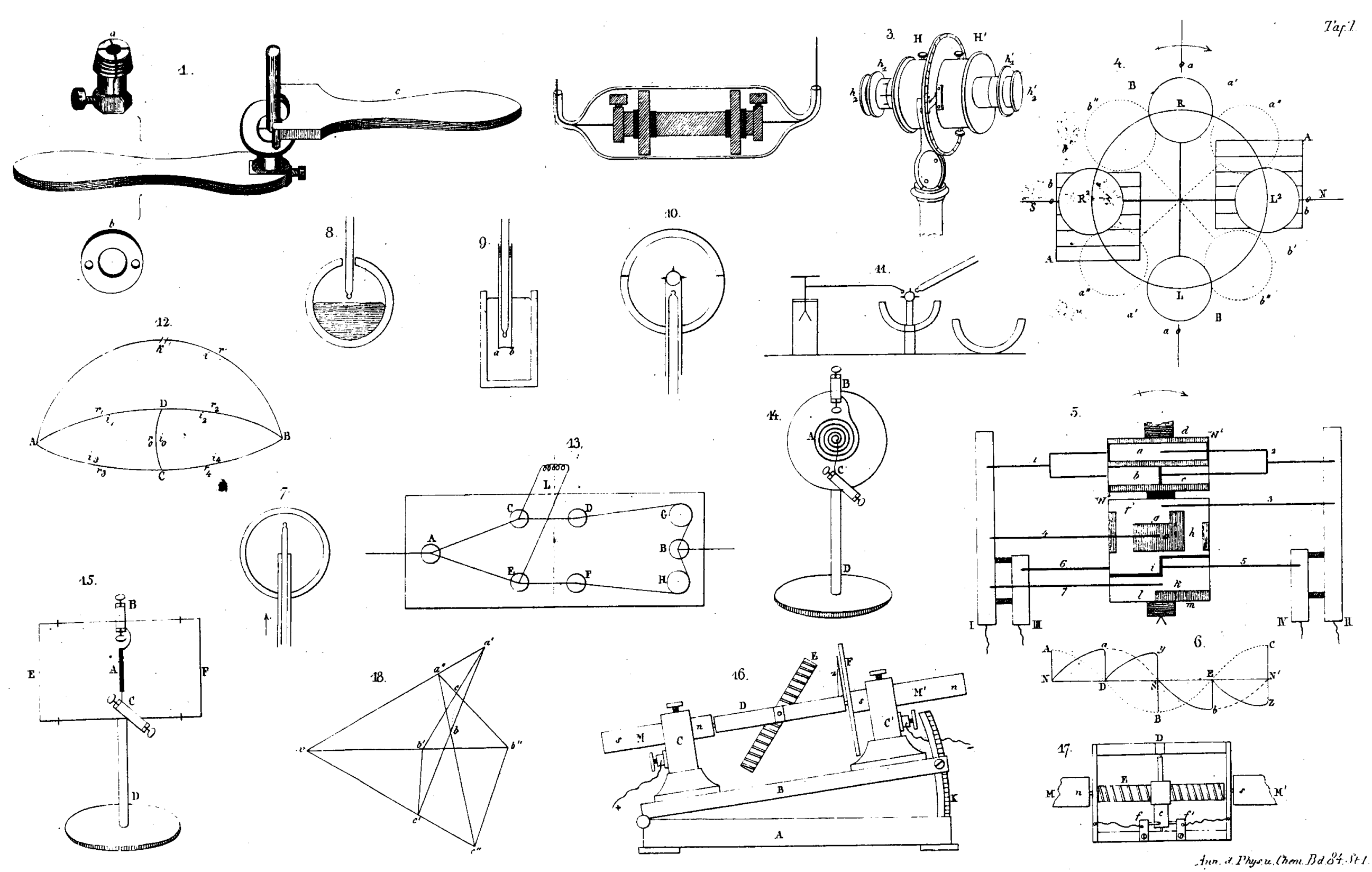

